\title{
Guerra Civil en El Salvador (1980-1992): análisis de las causas socio-estructural y la actuación de las Naciones Unidas
}

The Civil War in El Salvador (1980-1992): analysis of social-structure causes and the operation of the United Nations

Cinthya Janelle Milagros Celis Falcon ${ }^{1}$

\begin{abstract}
RESUMEN
El objetivo principal es el análisis del conflicto, intentando profundizar sobre las causas socio-estructurales y las acciones de paz de las Naciones Unidas durante el proceso de negociación. Se concluye que el Acuerdo de Paz, sólo termina formalmente con el enfrentamiento armado y no necesariamente con el conflicto social. Pues este, dio solución a problemas esencialmente institucionales con enfoque en el Estado.
\end{abstract}

Palabras-clave: Guerra Civil en el Salvador; ONUSAL; conflictos prolongados.

\begin{abstract}
The main objective is the analysis of the conflict, trying to deepen the structural causes and peacekeeping of UN during the negotiation process. It is concluded that the peace agreement, formally ending only with the armed confrontation and not necessarily with social conflict. Because, this solution was essentially for institutional problems with focus on the state.
\end{abstract}

Key-words: Civil War in El Salvador; ONUSAL, protracted conflicts.

\section{Edwar Azar y la Teoría de los conflictos sociales prolongados}

En el análisis de este conflicto utilizaremos a Edwar Azar (1990) teniendo como referencia el libro denominado: The Management of Protracted Social Conflict Theory and Cases. Asi, para el autor los conflictos prolongados incuban y crecen mayormente en lugares subdesarrollados. Los factores de estos pueden ser identidades religiosas, culturales o nacionalidad, ligados a las satisfacciones de las necesidades básicas como la seguridad, acceso justo a las instituciones políticas y a la justicia.

\footnotetext{
${ }^{1}$ Estudiante de Relaciones Internacionales e Integración en la Universidad Federal de Integración Latinoamericana (UNILA), Foz do Iguaçu, Brasil. 
Para Azar (1990), existen condicionantes que provocan un conflicto. Estos son: el contenido comunal, la satisfacción de las necesidades humanas, gobernabilidad y el rol del Estado, y las vinculaciones internacionales. Siendo que esta última tiene carácter de pre-condicionante de un conflicto social prolongado.

La propuesta del autor, es interesante no solo porque teoriza nuevos elementos que deberían ser analizados para entender una guerra no convencional, entre dos o más países. Sino porque apunta a que las causas de las guerras contemporáneas se pueden encontrar dentro del Estado-nación y fuera de ello, de manera que ambas estén supeditados uno al otro. El contenido comunal se explica:

\begin{abstract}
If a society is characterized by multicommunal composition, protracted social conflicts are most likely to arise. There are two factors which are responsible for the rise of politically active multicommunal societies. The first is a colonial legacy. Application of the principle of divide and rule by colonialists produced a unique political landscape in many parts of the world where a state artificially incorporated a multitude of communal groups. The second factor is a historical pattern of rivalry and contest among communal actors. (AZAR, 1990, p. 7, grifo nuestro)
\end{abstract}

La multiplicidad de las identidades/sociedades se da por dos factores esenciales, según Azar: la herencia colonial, originada en la conquista y establecida aun después de la independencia, como: la monopolización del poder estatal y económico; y por el histórico de rivalidades entre esas sociedades acentuada durante la colonización. Siendo que dichos factores provocan una relación no pacífica dentro de un Estado, debido a la desarticulación entre la sociedad como un todo y el Estado constituido con la colonización.

Con respecto a las necesidades humanas, Azar (1990, p. 7) alega que "we assume that individuals strive to fulfill their developmental human needs through the formation of identity groups. The most obvious ontological need is individual and communal physical survival and well-being. (grifo nuestro)". Dichas necesidades no son posibles por la falta de acceso a instituciones politicas, sociales, identidades culturales y distribución economica.

Por otro lado, la gobernabilidad y el rol del Estado, apunta al diseño del Estado y como ese aparato sirve para provocar y estar inserido en el conflicto. Eso ocurre cuando este está monopolizado por un grupo identitário dominante, que consecuentemente solo responde a la maximización de sus intereses individuales. Por lo tanto, provoca un 
despojo de la satisfacción de las necesidades básicas a una parte de la sociedad: no prevé seguridad ni desarrollo igualitario y suprime a aquellas sociedades de identidades diversas. De tal manera, que el tipo de gobierno o régimen gubernamental están vinculados a la dilatación del conflicto.

Por fin, las vinculaciones internacionales se expresa en la dependencia económica y en la relación clientelar política y militar con Estados fuertes. Estas acciones limitan la autonomía estatal en la toma de decisiones internas y direccionan sus acciones. Este último condicionante se entiende mejor con la inserción del país en un determinado contexto internacional y hasta regional.

Así mismo, es necesario tener en cuenta para el análisis de un conflicto prolongado dado el tiempo de duración estos generan deterioración de la seguridad física, el cual institucionaliza el subdesarrollo a través de la destrucción de las infraestructuras físicas y sociales. El debilitamiento institucional del aparato gubernamental, en los ámbitos socio-económicos y políticos, aumentando la poca interacción cooperativa y las divisiones comunales. La osificación psicológica, que en palabras de Azar (1990, p 17) protacted social conflict entails a vicious cycle of fear and hostile interactions among the communal contestants. Y el incremento de la dependencia y el clientelismo con países externos.

\section{Análisis de la guerra civil en El Salvador: Causas del conflicto}

Guillermo M. Ungo, salvadoreño de nacimiento, contribuye a entender las raíces del conflicto en El Salvador. Debido a que su artículo es publicado durante el desarrollo de la guerra. Este aporte lo realiza en un paper de título: Causas y perspectivas de la guerra civil en El Salvador (1984). No obstante para este artículo se desarrollaran tres de las causas mencionadas ${ }^{2}$, ya que tienen una base sólida explicativa.

\footnotetext{
${ }^{2}$ Entre las mencionadas por G. Ungo están las estructuras injustas, violencia institucional, bandera del anticomunismo, Reagan apoya a un gobierno asesino y corrupto, dólares y armas contra un pueblo que lucha por justicia y paz, y la guerra popular busca incorporar a la población a una democracia real. Los motivos de esta selección están relacionados a algunas carencias metodológicas del autor, pues algunos de sus argumentos están basados predominantemente en ideologías que en ningún momento lo sustenta con hechos concretos, fragilizando su investigación. 
El primero es la violencia institucional. Se refiere a cuando los mecanismos gubernamentales actúan de modo que favorezcan y gobiernen para minorías en detrimento de la mayor parte de la población. Lo que genera el no acceso igualitario a los "canales de participación democrática, violación masiva y creciente de los Derechos Humanos" (UNGO, 1984, p. 143). Por otro lado, la bandera del anticomunismo, se remite a una causa externa propia de la coyuntura regional-mundial de la Guerra Fría, que impactó en el ámbito interno del país. De modo, que la preocupación principal era el combate al "enemigo interno" sobre la base de la nefasta teoría de la "seguridad nacional" (1984, p.144) por parte de la oligarquía y el ejército que consecuentemente aplicaba una solución militarista.

Por último, se encuentra el apoyo económico-militar de los Estados Unidos. Solo en los primeros años de iniciado la guerra aportó \$ 70 millones de dólares que fue aumentando paulatinamente hasta 1985. Para luego sufrir un corte económico lento durante los próximos años hasta el final del conflicto. En el siguiente cuadro se ejemplifica lo aseverado.

U.S. Military Aid to El Salvador

\begin{tabular}{ll}
\hline Year & Amount (in millions) \\
\hline 1982 & $\$ 70.1$ \\
1983 & $\$ 80$ \\
1984 & $\$ 195.3$ \\
1985 & $\$ 134.8$ \\
1987 & $\$ 110$ \\
1988 & $\$ 89$ \\
1993 & $\$ 74.2$ \\
1994 & $\$ 0.4$ \\
\hline
\end{tabular}

CUADRO 1- Fuente: KLARE Y ANDERSON (1996) apud PUGH 2009, p. 90

Del mismo modo, Robert Orr (2001) apunta las causas ya mencionadas y agrega otras de tipo estructural-interna que está relacionado con las condiciones y oportunidades sociales asimétricas ofrecidas a la población salvadoreña: 
It had five principal causes: El Salvador's high levels of poverty and inequality (50 percent of the urban population lived below the poverty line); Little political space for dissent; significant problems with land ownership; systematic human rights abuse by the government; and international patronage and support for both sides (the US supported the government and Cuba supported the rebels) (ORR, 2001, p. 155-158),

\section{Actores envueltos: aplicación del modelo}

Para explicar los actores con participación directa e indirecta en el conflicto, Hugh Miall, Oliver Ramsbotham y Tom Woodhouse (2005: p. 27) nos presentan un modelo metodológico para analisar los actores. En seguida, se presentará un cuadro (2) con el fin de dar a entender quienes son y como participan en el conflicto interno salvadoreño. Donde el Track (1) hace referencia a entidades y personas gubernamentales y/o inter-gubernamentales. El Track (2) envuelve personas no gubernamentales. Y por último, el Track (3) que lo conforman los individuos y grupos locales.

\begin{tabular}{|c|c|c|c|}
\hline & TRACK 1 & TRACK 2 & TRACK 3 \\
\hline \multirow{5}{*}{ ACTORES } & $\begin{array}{l}\text {-Gobierno de El Salvador. } \\
\text {-Fuerzas Armadas (Roberto D' } \\
\begin{array}{l}\text { Aubuisson). Escuadrones de la } \\
\text { muerte. }\end{array}\end{array}$ & \multirow[t]{5}{*}{$\begin{array}{l}\text {-Iglesia Católica } \\
\text { de El Salvador }\end{array}$} & \multirow[t]{5}{*}{$\begin{array}{l}\text {-Frente Farabundo } \\
\text { Martí de Liberación } \\
\text { Nacional (FMLN) }\end{array}$} \\
\hline & -Estados Unidos & & \\
\hline & $\begin{array}{l}\text {-Organización de las Naciones Unidas } \\
\text { (ONU) }\end{array}$ & & \\
\hline & $\begin{array}{l}\text { - Grupo de Países Amigos: México, } \\
\text { España, Venezuela y Colombia. }\end{array}$ & & \\
\hline & $\begin{array}{l}\text {-Grupo de Apoyo a la Contadora: } \\
\text { Argentina, Brasil, Perú y Uruguay. }\end{array}$ & & \\
\hline \multirow{5}{*}{$\begin{array}{l}\text { TECNICAS } \\
\text { UTILIZADAS }\end{array}$} & $\begin{array}{l}\text {-Represión coercitiva. } \\
\text {-Reclutamiento de niños como } \\
\text { soldados. }\end{array}$ & \multirow{5}{*}{ 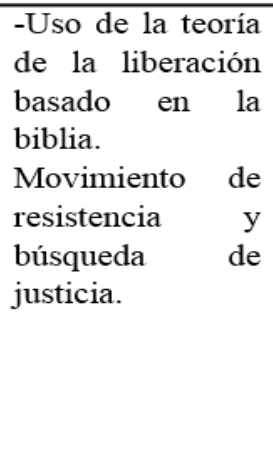 } & \multirow[t]{5}{*}{$\begin{array}{l}\text {-Estrategia de } \\
\text { guerrilla. Ubicación } \\
\text { en las zonas rurales. }\end{array}$} \\
\hline & $\begin{array}{l}\text {-Financiamiento económico-militar } \\
\text { para el gobierno. }\end{array}$ & & \\
\hline & $\begin{array}{l}\text {-Mediador, observador y negociador } \\
\text { del conflicto y constructor de la paz. }\end{array}$ & & \\
\hline & $\begin{array}{ll}\text {-Mediadores } & \text { durante } \\
\text { negociaciones. } & \end{array}$ & & \\
\hline & -Promoción de la paz y mediador. & & \\
\hline
\end{tabular}

CUADRO 2- ACTORES EN LA GUERRA CIVIL EN EL SALVADOR. Fuente: Elaboración propia. 
Dicho método, es una manera de clasificar a los actores. Eso no implica que su ubicación esté relacionada a su papel en el conflicto, porque su peso político, económico, etc., varía según el contexto en la que se insieren.

\title{
Sobre la Misión de Observadores de las Naciones Unidas en El Salvador
}

La Misión de Observadores de las Naciones Unidas en El Salvador (ONUSAL) tuvo una duración de cuatro años desde julio de 1991 hasta abril de 1995. Su sede estaba en San Salvador, capital de dicho país centroamericano. Javier Pérez de Cuellar como Secretario General de las Naciones Unidas (1982-1991) acompañó el proceso de negociaciones hasta el acuerdo definitivo de paz de la guerra civil en El salvador. Del mismo modo, el trabajo desempeñado por Álvaro Soto es destacable ya que fue el negociador en el conflicto entre el FLMN y el Estado salvadoreño.

En un primer momento, la función de la ONUSAL era "verificar la aplicación de todos los acuerdos que incluían una cesación del fuego y medidas conexas, la reforma y la reducción de las fuerzas armadas, la creación de un cuerpo de policía, la reforma de los sistemas judicial y electoral, los derechos humanos, la posesión de la tierra y otros asuntos económicos y sociales". De ahí que,

La ONUSAL se estableció en virtud de la resolución 693 (1991) del Consejo de Seguridad de 20 de mayo de 1991. Sus divisiones, establecidas en diferentes momentos del transcurso de la Misión, son: División de Derechos Humanos, Militar y de Policía. (Misiones de Observadores de las Naciones Unidas en El Salvador, online)

Además, estaba compuesta por:

\begin{abstract}
El primer Representante Especial del Secretario General y Jefe de Misión de la ONUSAL fue el Sr. Iqbal Riza (el Pakistán), que desempeñó su función hasta marzo de 1993. Le sucedió el Sr. Augusto Ramírez Ocampo (Colombia), que desempeñó su función hasta marzo de 1994, cuando le sucedió el Sr. Enrique ter Horst (Venezuela). (Misiones de Observadores de las Naciones Unidas en El Salvador, online)
\end{abstract}

Como se puede visualizar, las primeras acciones de la ONUSAL se concentraban en acciones burocráticas de restablecimiento del orden. Además, que hubo una predominancia de la participación de algunos representantes de América del Sur y 
Europa. Eso muestra que el conflicto en El Salvador no era considerado ajeno a la realidad latinoamericana, lo que les otorga protagonismo regional en las conversaciones de paz, inserido en un contexto de finales de la Guerra Fría.

\begin{abstract}
La misión ONUSAL contó con 380 observadores militares, ocho médicos y 631 observadores de policía; también participaron 140 civiles de contratación internacional y 180 más de contratación local. Tuvo un costo de 107 millones de dólares. Entre los militares, la misión fue encabezada por 15 oficiales de Canadá, Brasil, Ecuador, España y Venezuela. La división de policía fue dirigida por 16 funcionarios del ramo de España, Francia e Italia. En esta división participaron 120 policías mexicanos, además de elementos de Brasil, Chile, Colombia, Guyana, Italia, España, Suecia y Venezuela. (BENITEZ, 2007, p. 108)
\end{abstract}

Por otro lado, Holiday y Stanley (1993) en The Peace: preliminary lessons from El Salvador, califica a la ONUSAL de ser una de las misiones de paz de la cual se aprendió nuevas técnicas de negociación, debido a que este era un conflicto que no estaba caracterizado por una disputa territorial entre dos o más países, poniéndoles así desafíos aún mayores. Del cual se obtuvo resultados positivos por la multiplicidad de papeles que tuvo que desempeñar la Misión en un corto tiempo:

\begin{abstract}
This was the first time that the United Nations attempted to broker the end of an internal conflict; in the process, the international body developed a number of new approaches to peacemaking. The United Nations gradually expanded its role in the negotiations from that of observer, to active mediator and, ultimately, to verifier of the accords. (HOLIDAY; STANLEY, 1993, p.1)
\end{abstract}

De tal modo, dicha complejidad del conflicto llevo a que la misión de paz en El Salvador pase de un observador a un agente de construcción de la paz que incluyó acciones más profundas:

Agenda for Peace, peace-building efforts may include weapons seizure and destruction, restoration of order, refugee repatriation, training of security personnel, election monitoring, protection of human rights, reform of governmental institutions and promotion of political participation. (HOLIDAY;STANLEY, 1993, p.2) 


\section{Proceso de Negociación}

Para Ricardo Ribera (1994) en “El Salvador: La negociación del Acuerdo de Paz ¿un modelo para el mundo?" apunta que los motivos que llevaron, luego de 10 años de guerra, a los acercamientos concretos del proceso de paz estuvieron relacionados al agotamiento social. Así, para el FMLN permanecer unidos en una estrategia continua de guerra provocaba un debilitamiento en la agrupación. Lo que llevaba a una perdía de confianza y aumentando el cansancio de la sociedad salvadoreña sobre la violencia suscitada. Sumado a ello, se encontraba el movimiento pacificador de los representantes de la iglesia católica a través de la teoría de la liberación. Este además de ser una muestra de resistencia simbolizaba un basta a los abusos de la guerra. A continuación se desarrollará, de manera sintética, los cinco principales acuerdos en el proceso de paz en base a Ricardo Ribera (1994), dando énfasis a las acciones de paz de las Naciones Unidas.

Acuerdo de Ginebra marcó el inicio oficial del proceso de negociación el 4 de abril de 1990 en la sub sede de la ONU. Luego de que en noviembre de 1989 el FMLN mostró su fuerza bélica logrando impactar en la opinión pública mundial. Y de que el gobierno mostrara un debilitamiento en cuanto a la constancia de sostener una estrategia de contención a la guerrilla. Dando motivos suficientes para dar paso al dialogo entre las partes. El encuentro tenía cuatro propósitos: poner fin al conflicto armado por la vía política, democratización del país, respeto a los DD.HH y la reunificación de la sociedad. Los objetivos de la negociación era alcanzar un acuerdo político y establecer garantías y condiciones de reincorporación del FMLN a la vida política e institucional.

Acuerdo de Caracas,suscitada el 21 de mayo de 1990, se fija la agenda y el calendario de negociación. En la primera fase el gobierno tendría que responder por seis puntos de estructuración institucional: Fuerza Armada (FF.AA.) DD.HH, sistema judicial, sistema electoral, reforma constitucional y problema económico-social. Para dar paso a la segunda fase, se tenía que cumplir la primera; luego el FMLN tendría la posibilidad de inserirse de forma legal como un partido para disputar por las elecciones de marzo de 1991. Todo esto, estaba propuesto alcanzarlo a mediados de setiembre de 1990. 
Acuerdo de San José, desde el comienzo se tomó como tema principal las FF.AA, no obstante este tema creó un ambiente de tensión entre las partes que tenían posiciones diferentes al respecto. Ante ello, el representante personal del Secretario General de la ONU, el diplomático peruano, Álvaro Soto propuso que se dejara por en cuanto de lado el tema de las FF.AA. y dedicarle tiempo al punto sobre DD.HH. Propuesta que resulto favorable ya que como producto salió el Convenio sobre los DD.HH, compromiso cerrado el 26 de julio de 1990. Lo que se planteaba en dicho documento era garantizar el respeto a los derechos humanos y establecer una “Misión” de la ONU que vigile su cumplimiento; que más tarde en 1991, se denominará como ONUSAL.

Acuerdo de New York fue convocado por el Secretario General, Pérez de Cuellar en la sede de la ONU para el 16 al 17 de setiembre de 1991, con el fin de dar solución al problema de las fuerzas militares de ambas partes. Esta reunión significó el acuerdo de compromisos decisivos, ya que que se conformó una Comisión ad hoc: encargada de la reducción de las fuerzas armadas y de la creación de la Policía Nacional Civil. Así como, la creación de la Comisión Nacional para la Consolidación de la Paz (COPAZ). Ante ello, el Consejo de Seguridad de la ONU mostró apoyo al proceso de paz, así, lo hicieron también el Grupo de Países Amigos: México, España, Venezuela y Colombia.

Acuerdo de Chapultepec (México), según Ribera (1994, p. 127) para el comandante del FMLN, Schafik Handal el Acuerdo de Paz en Chapultepec era "expresión del nuevo consenso de la Nación" y para el presidente de El Salvador Cristiani era símbolo del fortalecimiento de la democracia en el país. Lo cierto es que con el Acuerdo de Paz firmado en Chapultepec el 16 de enero de 1992, se daba un aparente fin a la hegemonía militar sobre la nación civil (1994, p.128), exclamado por Schafik Handal. Es decir, él estaba haciendo referencia a conformación de un estado justo y menos represivo, en la cual las fuerzas armadas estén al servicio del pueblo y no contra la sociedad salvadoreña. 


\section{Paradigma de la inseguridad provocada por los Maras: Pos - guerra civil salvadoreña}

Según Jaime Matinez (2012, p.2) las acciones de los maras se ampliaron desde finales de la guerra civil (1992). Pues se produjo un proceso de deportación y regreso de la población salvadoreña hacia el país, originándose una transculturación entre los que se quedaron durante la guerra y los que huyeron. Aunque el tema sobre los maras sea muy compleja y polémico por su relación con el crimen organizado, se debe tener en cuenta la multiplicidad de los factores causales que lo provocan. En tal sentido, que haciendo una relación de comparación entre las causas que originaron la guerra civil y los factores originarios de los maras, se encuentra vínculos en cuanto a factores sociales, económicos, políticos e institucionales.

\begin{tabular}{|c|c|c|c|c|}
\hline Factores soxiales & $\begin{array}{l}\text { Factores } \\
\text { economicos }\end{array}$ & $\begin{array}{l}\text { Factores politicose } \\
\text { institusionales }\end{array}$ & $\begin{array}{l}\text { Factores } \\
\text { culturales }\end{array}$ & $\begin{array}{l}\text { Factores } \\
\text { gircunstandiales } 0 \\
\text { habilitadores }\end{array}$ \\
\hline $\begin{array}{l}\text { linsatisfaccion de } \\
\text { derechosisociales } \\
\text { basicos }\end{array}$ & $\begin{array}{l}\text { Desigualdad } \\
\text { economical }\end{array}$ & $\begin{array}{l}\text { Educad on pobre y de } \\
\text { poca cobertura }\end{array}$ & $\begin{array}{l}\text { Aceptaoonisocial } \\
\text { delavolencia }\end{array}$ & $\begin{array}{l}\text { Proliferacion y fácl } \\
\text { acceso a las armas } \\
\text { de fuego }\end{array}$ \\
\hline $\begin{array}{l}\text { Problemas heredados } \\
\text { de conflictos belicos } \\
\text { anteriores. }\end{array}$ & $\begin{array}{l}\text { Empleos precanios } \\
\text { explotadoresy } \\
\text { continuadores de la } \\
\text { pobreza. }\end{array}$ & $\begin{array}{l}\text { Falta de programas: } \\
\text { educativos adecuados }\end{array}$ & Transculturacion & $\begin{array}{l}\text { Conodimientos y, } \\
\text { tecnologla heredada } \\
\text { de conflictos belicos }\end{array}$ \\
\hline $\begin{array}{l}\text { Emigradion del } \\
\text { campo alla oudad }\end{array}$ & $\begin{array}{l}\text { Falta de empleos } \\
\text { para jovenes }\end{array}$ & $\begin{array}{l}\text { Sistema educativo } \\
\text { intolerantey exulsivo }\end{array}$ & $\begin{array}{l}\text { Machismogy elogo } \\
\text { de la matonena }\end{array}$ & $\begin{array}{l}\text { Narcotrafico yg abuso } \\
\text { de alcoholy drogas }\end{array}$ \\
\hline $\begin{array}{l}\text { Altaconcentración } \\
\text { poblaconal }\end{array}$ & $\begin{array}{l}\text { Emigradón } \\
\text { economical }\end{array}$ & Desercion escolar & $\begin{array}{l}\text { Eraltacionidelal } \\
\text { astuciagy la falta } \\
\text { de escrupulos para } \\
\text { conseguir lo que se } \\
\text { gulere. }\end{array}$ & $\begin{array}{l}\text { Escasez de espacios: } \\
\text { recreativosi } 0 \\
\text { deportivos: }\end{array}$ \\
\hline $\begin{array}{l}\text { Vivendas } \\
\text { inadecuadas, } \\
\text { urbanizadion } \\
\text { descrdenada }\end{array}$ & $\begin{array}{l}\text { Falta de } \\
\text { oportunidades, para } \\
\text { superar la pobreza }\end{array}$ & $\begin{array}{l}\text { Corrupcion } \\
\text { instituoonal }\end{array}$ & $\begin{array}{l}\text { Paternidady } \\
\text { matemidad } \\
\text { irresponsable }\end{array}$ & $\begin{array}{l}\text { Influencias } 0 \\
\text { presiones de grupos! } \\
\text { de amigos }\end{array}$ \\
\hline Familias debfitadas & $\begin{array}{l}\text { Marginalidadiy } \\
\text { exdursion sociall }\end{array}$ & $\begin{array}{l}\text { Debfilidad yffalta } \\
\text { deconfianzaren las } \\
\text { instituoiones. }\end{array}$ & $\begin{array}{l}\text { Intolerancia hacia } \\
\text { quilenes sonivistos, } \\
\text { como differentes. }\end{array}$ & $\begin{array}{l}\text { linodencia de } \\
\text { los.medios de } \\
\text { comunicacion }\end{array}$ \\
\hline $\begin{array}{l}\text { Falta de } \\
\text { Gportunidades, de } \\
\text { [inserobingocial }\end{array}$ & $\begin{array}{l}\text { Pollibicas } \\
\text { inediberales } \\
\text { quereducen la } \\
\text { inversióngociall }\end{array}$ & $\begin{array}{l}\text { Impunidad de la } \\
\text { criminalidad compleja } \\
\text { ode cuello blanco }\end{array}$ & $\begin{array}{l}\text { Programacoin } \\
\text { televisivaviblentayg } \\
\text { exaltadora de vicies }\end{array}$ & $\begin{array}{l}\text { Ausencia rescasez: } \\
\text { devalores eticosy } \\
\text { moralles }\end{array}$ \\
\hline $\begin{array}{l}\text { Falta de liderazgos } \\
\text { rejemplares }\end{array}$ & & $\begin{array}{l}\text { Roliticacciminal } \\
\text { gelectiva y } \\
\text { prindipalmente } \\
\text { punitiva }\end{array}$ & & $\begin{array}{l}\text { Decibiones, } \\
\text { iindividuales. }\end{array}$ \\
\hline
\end{tabular}

CUADRO 3- FACTORES RELACIONADOS CON ELFENOMENOS DE LAS MARAS O PANDILLAS.

FUENTE: MARTINEZ (2012, p. 3) 
En este sentido, se llama la atención, solo a modo de ejemplo, a los factores sociales como: la insatisfacción de los derechos sociales, falta de oportunidades de inserción social y problemas heredados de la guerra. A factores económicos, empleos precarios y continuadores de la pobreza, marginalidad y exclusión social. A factores políticos: debilidad y falta de confianza en las instituciones. A factores culturales: aceptación social de la violencia; y a factores circunstanciales como el fácil acceso a armas de fuego. Quiere decir, que el surgimiento de las maras está ligado a la guerra civil en El Salvador, específicamente a aquellas causas que llevaron al conflicto o que emergieron durante las disputas y que la ONUSAL en teoría resolvió con el Acuerdo de Chapultepec.

\section{Conclusiones}

Se concluye que los aportes conceptuales de Azar (1990) sobre las necesidades humanas individual o colectiva tuvieron una correlación directa con el de gubernamentabilidad y el papel del Estado. Pues, dado el caso que se analizó la elite hizo del Estado un instrumento de materialización para la satisfacción de sus intereses, el cual provocó escasez, en las clases no terratenientes, de bienestar y justicia social. Se deja claro también, que las vinculaciones internacionales fueron una fuerza importante de impulso del conflicto.

Con el acuerdo de Paz en 1992 se daba inicio al fin del conflicto político-militar armado entre el FMLN y el gobierno de El Salvador. Sin embargo, en base a Azar (1990) los conflictos sociales prolongados no se agotaron, al contrario tomaron nuevas formas de expresión que generaron inseguridad interna en la población salvadoreña. Que son frutos de la falta de efectividad concreta de las misiones de paz para dar respuestas durables y profundas ante las verdaderas causas social-estructurales. Pues las medidas tomadas fueron direccionadas a solucionar problemas políticos-institucionales y no necesariamente sociales. Pero aun aquellas medidas institucionales con el tiempo no se mostraron sólidas, ejemplo de ello son las maras. A esto se agrega el poco compromiso real asumido pos- negociaciones de paz, por el gobierno y por el FMLN.

Es decir, los acuerdos de paz de enero de 1992 acabaron con la guerra y con el enfrentamiento físico, pero no con las condiciones socio-económicas que la generaron y 
que siguen promoviendo tanta muerte injusta. El alcance de paz es mucho más que el final de un conflicto armado. Dado que el gobierno de El Salvador sigue comprometido a fondo con un modelo de país que ahonda las desigualdades sociales. Ahí está la raíz estructural de la violencia y mientras no se desarraigue esta "guerra económica-social" muchas otras violencias y exclusiones mantendrán sin paz a los salvadoreños.

Por otro lado, la mediación de la ONU es considerada el esfuerzo más exitoso de todos en los que se ha involucrado para solucionar conflictos similares. De los cinco conflictos en los que la ONU ha invertido su capital político y diplomático en los dos últimos años [anterior a 1993] - Camboya, Angola, Somalia, Yugoslavia y El Salvador sólo este último registra saldo positivo (BENITEZ, 1993, p. 10).

Así mismo, queda a reflexión hasta qué punto la comisión de Derechos Humanos $\mathrm{u}$ otras instituciones que fueron creadas para sanar las heridas de la guerra civil salvadoreña tocaron el tema sobre el uso de niños soldados y la preparación de los mismos tanto por el FMLN y como por el gobierno. Ya que por parte del primero, el reclutamiento de los niños se daba de forma voluntaria y por el contacto que la familia tenía con la guerrilla. En cambio, para el segundo, este se daba a partir de los 12 años y era de carácter obligatorio. Niños que eran preparados para luchar contra las guerrillas sobre todo en las zonas rurales. Además, aquellos niños se caracterizaban porque eran mayoritariamente de lugares donde se instalaba el FMLN, de condición económica precaria y de fácil vulnerabilidad ideológica.

\section{REFERENCIAS}

ACUERDO DE CHAPULTEPEC. Ciudad de México, 16 de enero de 1991.

AZAR, Edward. The Management of Protracted Social Conflict: Theory and Cases. Aldershot: Darthmouth Publishers, 1990. P. 1-17

BELlAMY, Alex J.; WILlIAMS, Paul D. United Nations Observer Mission in El Salvador (ONUSAL). Understanding Peacekeeping. Publicado el 20 de enero del 2010. Visualizado el 01 de noviembre de 2014

BENITEZ, Raúl. El Salvador. Paz conflictiva, Democracia frágil. Nueva Sociedad no․126, p. 6-10, julio- agosto de 1993. 
BENITEZ, Raúl. América Latina: Operaciones de Paz y acciones militares internacionales de las Fuerzas Armadas. Foro Internacional. Vol. XLVII, p. 99-116, enero-marzo del 2007.

Equipo Envío. El Salvador: Acuerdo de New York. Revista Envio, Nicaragua, no 120 , Oct. 1991.

Equipo Envío. El Salvador: La extrema derecha desafía a la ONU. Revista Envio,

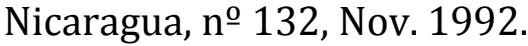

GALTUNG, Johan. Violence, Peace, and Peach Research. Journal of Peace Research, 6 (3), 1969, p. 167-191.

HOLIDAY, David; STANLEY, William. Building the Peace: preliminary lessons from El Salvador. Journal of International Affairs, vol. 46. 1993

KRSTICEVIC, Viviana; HERNÁNDEZ, María J. El Salvador: Monseñor Romero: crimen impune, caso abierto. Revista Envio, Nicaragua, no 216, marzo de 2000.

MARTINEZ, Jaime. Maras en El Salvador y su relación con el crimen organizado transnacional. p. 1-12, noviembre del 2010,

MIALL, Hugh; RAMSBOTHAM, Oliver; and WOODHOUSE, Tom. Introduction to Conflict Resolution: Concepts and Definitions. In: Contemporary Conflict Resolution: The Prevention, Management and Transformation of Deadly Conflicts. Cambridge: Polity Press, 2005.

MORENO, Ismael. El Salvador: La subversiva memoria de todos los mártires. Revista Envio, Nicaragua, ํㅡ 213, diciembre de 1999.

ORGANIZACIÓN DE LAS NACIONES UNIDAS. Misiones de Observadores de las Naciones Unidas en El Salvador (ONUSAL). Disponible en: http://www.un.org/es/peacekeeping/missions/past/onusal.htm. Acceso el 01 de noviembre del 2014

ORR, Robert C. Building Peace in El Salvador: From Exception to Rule. In: Cousens and C. Kumar (eds.). Peacebuilding as Politics. Estados Unidos de America: Lynne Rienner Publishers, 2001. p. 153-181.

PUGH, Jeffrey. The Structure of Negotiation: Lessons from El Salvador for Contemporany Conflict Resolution. Negotiation Journal, p. 83-105, 2009

RIBERA, Ricardo. El Salvador: La negociación del Acuerdo de Paz. ¿un modelo para el mundo?. Realidad (37), p. 89-134, enero-febrero de 1994.

SERRANO, Omar. El Salvador: Campaña pre-electoral: ¿y los acuerdos de paz?. Revista Envío, Nicaragua, no 138, junio de 1993.

UNGO, Guillermo M. Causas y perspectivas de la guerra civil en El Salvador. Revista Mexicana de Sociología. Vol. 46, nº 3, p. 143-154, julio-setiembre de 1984.

Voces inocentes. Disponible en: https://www.youtube.com/watch?v=0W3Wil0Hta4. Visualizado el 31 de octubre del 2014 